\title{
Possible Neuroinflammatory Mechanisms Which May Lead to Long-Term Neurological Disorders in COVID-19 Patients
}

\author{
Michael Lange ${ }^{1}$, Riyi Shi ${ }^{2}$ \\ ${ }^{1}$ Indiana University School of Medicine, ${ }^{2}$ Purdue University, Department of Basic Medical \\ Sciences
}

This review aims to provide insight into the possible long-term neurological complications that COVID-19 patients may experience after the resolution of intense acute inflammation characterized as "cytokine storm." Neurological symptoms such as fatigue, headache, dizziness, nausea, confusion, dyspnea, anorexia, malaise, myalgia, ataxia, seizure, hypogeusia, and hyposmia are commonly seen in these patients. COVID-19 related encephalitis is also seen sporadically. However, some researchers believe neuroinflammation is more common than what is reported. Neurological abnormalities that are linked to neuroinflammation are of particular concern because neuroinflammation is hypothesized to cause neurological diseases such as Alzheimer's Disease, Parkinson's Disease, and Schizophrenia. Many potential routes can lead to inflammation in the nervous system and elicit neuron cell death in COVID-19 patients. These include the potential neurotropic pathway of the novel coronavirus, CNS parenchymal infectability, thrombotic ischemic stroke, cytokine storm, and blood-brain barrier breakdown. Past pandemics of similar neurotropic viruses could also offer insights regarding the long-term neurological effects of COVID-19. In support of our hypothesis, the Spanish Flu pandemic of 1918-1919 saw an increased incidence of neurodegenerative disease, Parkinson's disease, and schizophrenia. We do not know exactly what the future will hold for COVID-19 however, it is of paramount importance to attempt to anticipate and prepare for the possible chronic neurological sequelae and mitigate or prevent their effects. 